\title{
Long- and short-range magnetism in the frustrated double perovskite $\mathrm{Ba}_{2} \mathrm{MnWO}_{6}$
}

\author{
Heather Mutch, Otto Mustonen, Helen C. Walker, Peter J. Baker, \\ Gavin B. G. Stenning, Fiona C. Coomer, and Edmund J. Cussen
}

\section{Published version information}

Citation: $\mathrm{H}$ Mutch et al. "Long- and short-range magnetism in the frustrated double perovskite $\mathrm{Ba}_{2} \mathrm{MnWO}_{6} . "$ Physical Review Materials, vol. 4, no. 1 (2020): 014408.

DOI: $10.1103 /$ PhysRevMaterials.4.014408

This version is made available in accordance with publisher policies. Please cite only the published version using the reference above. This is the citation assigned by the publisher at the time of issuing the APV. Please check the publisher's website for any updates. 


\title{
Long- and short-range magnetism in the frustrated double perovskite $\mathrm{Ba}_{2} \mathrm{MnWO}_{6}$
}

\author{
Heather Mutch, ${ }^{1}$ Otto Mustonen $\odot,{ }^{1}$ Helen C. Walker, ${ }^{2}$ Peter J. Baker $\odot,{ }^{2}$ Gavin B. G. Stenning, ${ }^{2}$ \\ Fiona C. Coomer ${ }^{3},{ }^{3}$ and Edmund J. Cussen ${ }^{1}{ }^{1, *}$ \\ ${ }^{1}$ Department of Material Science and Engineering, University of Sheffield, Mappin Street, Sheffield S1 3JD, United Kingdom \\ ${ }^{2}$ ISIS Pulsed Neutron and Muon Source, STFC Rutherford Appleton Laboratory, Harwell Campus, Didcot OX11 OQX, United Kingdom \\ ${ }^{3}$ Johnson Matthey Battery Materials, Blount's Court, Sonning Common, Reading RG4 9NH United Kingdom
}

(Received 9 October 2019; published 27 January 2020)

\begin{abstract}
The structural and magnetic properties of the face-centered-cubic double perovskite $\mathrm{Ba}_{2} \mathrm{MnWO}_{6}$ were investigated using neutron powder diffraction, DC magnetometry, muon spin relaxation, and inelastic neutron scattering. $\mathrm{Ba}_{2} \mathrm{MnWO}_{6}$ undergoes type II long-range antiferromagnetic ordering at a Néel temperature of $8(1)$ $\mathrm{K}$ with a frustration index, $f \approx 8$. Inelastic neutron scattering was used to identify the magnetic coupling constants $J_{1}$ and $J_{2}$, which were found to equal -0.080 and $-0.076 \mathrm{meV}$, respectively. This indicates that both of the magnetic coupling constants are antiferromagnetic with similar magnitudes, which is in contrast to other known $3 d$ metal double perovskites of the form $\mathrm{Ba}_{2} M \mathrm{WO}_{6}$. Above the Néel temperature, muon spin-relaxation measurements and inelastic neutron-scattering techniques identify a short-range correlated magnetic state that is similar to that observed in the archetypical face-centered-cubic lattice antiferromagnet $\mathrm{MnO}$.
\end{abstract}

DOI: 10.1103/PhysRevMaterials.4.014408

\section{INTRODUCTION}

Geometrical frustration is a phenomenon which can prevent antiferromagnetic systems from achieving a unique ground state. This is driven purely by the structure of the material and primarily affects lattices which consist of connecting triangular plaquettes, such as planar kagome lattices, pyrochlores, and the face-centered cubic (fcc) structure [1]. Geometrical frustration can result in a number of novel states such as spin glass [2,3], spin ice [4,5], spin liquids [6,7], and other complex states such as the valence bond glass $[8,9]$. Frustration has also been shown to cause unexpected behavior above the Néel temperature $\left(T_{N}\right)$, where short-range correlations can take place $[10,11]$. Many of these novel spin states are found when magnetic cations are arranged in the fcc lattice and we have undertaken an extensive study of these frustration effects.

Magnetism in fcc lattice compounds can often be described using a simple $J_{1}-J_{2}$ Heisenberg model:

$$
\hat{H}=-J_{1} \sum_{\langle i j\rangle} S_{i} \cdot S_{j}-J_{2} \sum_{(i j)} S_{i} \cdot S_{j},
$$

where $J_{1}$ is the nearest-neighbor (NN) interaction, $J_{2}$ is the next-nearest-neighbor (NNN) interaction, $S_{i}$ is spin at site $i$, and the sums are taken over all $\mathrm{NN}\left(J_{1}\right)$ and $\mathrm{NNN}\left(J_{2}\right)$ interactions, respectively. Using the above equation, antiferromagnetic interactions are denoted using $J<0$ while $J>0$ for ferromagnetic interactions. Three distinct antiferromagnetically ordered ground states are possible depending on the relative strengths of the $J_{1}$ and $J_{2}$ interactions: type I, type II, and type III. Type II antiferromagnetic order is the

\footnotetext{
*e.j.cussen@sheffield.ac.uk
}

most commonly observed magnetic structure in fcc lattices. The type II structure occurs when $J_{2}$ is dominant and antiferromagnetic, and as a result all next-nearest-neighbor magnetic cations couple antiferromagnetically. In this instance, magnetic frustration arises from the weaker $J_{1}$ interaction as not all nearest-neighbor magnetic cations are able to couple antiferromagnetically [12,13].

$\mathrm{MnO}$ is an archetypal type II fcc antiferromagnet and has been shown to experience frustration effects. In 1949, Shull and Smart reported the detection of antiferromagnetic peaks at $80 \mathrm{~K}$ in $\mathrm{MnO}$ using neutron diffraction. This was the first time this signature of antiferromagnetism was directly observed and $\mathrm{MnO}$ was correctly identified as possessing the propagation vector $\boldsymbol{k}=(1 / 2,1 / 2,1 / 2)$ [14]. Since this was reported $\mathrm{MnO}$ has been keenly investigated and was initially reported to experience a structural phase transition from $\mathrm{cu}-$ bic to rhombohedral at its magnetic transition temperature of $118 \mathrm{~K}$. This transition was stated to enable long-range antiferromagnetic order to take place within $\mathrm{MnO}$, where $\mathrm{Mn}^{2+}$ cations are arranged in a parallel configuration in (111) planes and any perpendicular (111) planes are arranged in an antiparallel manner $[15,16]$. However, this is now known to be the result of a monoclinic distortion to space group $C 2$, rather than a rhombohedral distortion [17]. More recently, $\mathrm{MnO}$ has also been reported to exhibit a short-range correlated state above its transition temperature, which is thought to be the result of magnetic frustration within the system [18].

As well as simple oxides such as $\mathrm{MnO}$, double perovskites such as $\mathrm{Ba}_{2} \mathrm{MnWO}_{6}$ also host magnetic cations on an fcc lattice. Like $\mathrm{MnO}, \mathrm{Ba}_{2} \mathrm{MnWO}_{6}$ has a magnetic structure composed of high-spin $\mathrm{Mn}^{2+}$ cations $\left(3 d^{5}, S=5 / 2\right)$. $\mathrm{Ba}_{2} \mathrm{MnWO}_{6}$ crystallizes in the space group $F m \overline{3} m$, remaining cubic until $2 \mathrm{~K}$ (Fig. 1), with no structural phase transitions reported. This was initially studied in 1976 by Khattak et al., who reported 


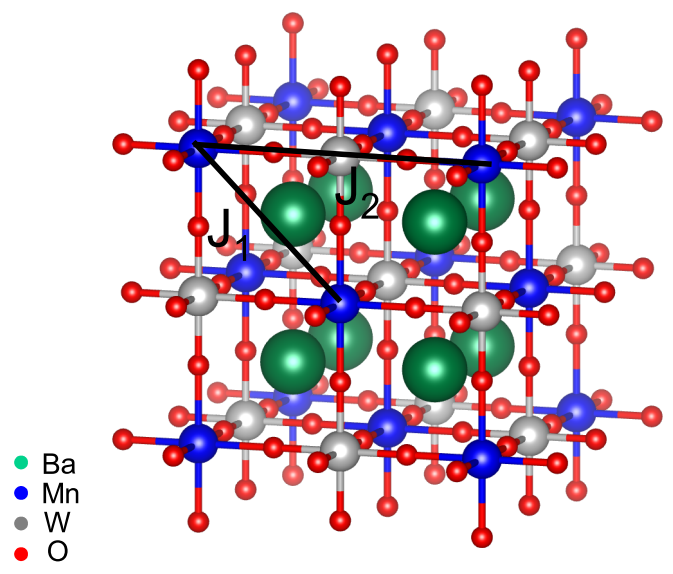

FIG. 1. Crystallographic structure of $\mathrm{BaMnWO}_{6}$ with the magnetic exchange interactions $J_{1}$ and $J_{2}$ displayed.

a type II antiferromagnetic structure with propagation vector $\boldsymbol{k}=(1 / 2,1 / 2,1 / 2)$ and a magnetic transition temperature of approximately $9 \mathrm{~K}$. This was based on neutron-powder diffraction and DC-magnetometry measurements [19]. Further studies completed on $\mathrm{Ba}_{2} \mathrm{MnWO}_{6}$, correlated fairly well with this initial assessment, also indicating a type II antiferromagnetic (AFM) structure [20]. However, the possibility of short-range magnetic correlations similar to those present in $\mathrm{MnO}$ has not been investigated nor are the magnetic interactions $J_{1}$ and $J_{2}$ known.

This study presents a comprehensive investigation of the magnetic behavior observed in $\mathrm{Ba}_{2} \mathrm{MnWO}_{6}$ utilizing neutronpowder diffraction, DC magnetometry, muon spin relaxation, and inelastic neutron scattering. Using this range of techniques, we have characterized the type II antiferromagnetic order in $\mathrm{Ba}_{2} \mathrm{MnWO}_{6}$ below a transition temperature of $8(1) \mathrm{K}$. Above this transition temperature, a short-range correlated state, similar to that described in $\mathrm{MnO}$ [18], was detected using muon spin relaxation in conjunction with inelastic neutron scattering. Inelastic neutron scattering shows that the magnetic interactions $J_{1}$ and $J_{2}$ are both antiferromagnetic with almost equal magnitude, with $J_{1}$ the slightly stronger coupling constant. This is an unexpected trait of $\mathrm{Ba}_{2} \mathrm{MnWO}_{6}$ which is not observed in other similar double perovskites and is interesting as $J_{2}$ interactions remain unfrustrated within the magnetic lattice.

\section{EXPERIMENTAL}

A powder sample of $\mathrm{Ba}_{2} \mathrm{MnWO}_{6}$ was prepared via solidstate synthesis using stoichiometric quantities of $\mathrm{BaCO}_{3}$, $\mathrm{MnO}_{2}$, and $\mathrm{WO}_{3}$. The reagents were mixed with extensive grinding and calcined in air at $800^{\circ} \mathrm{C}$. The powder was then thoroughly ground, pressed into 13 -mm-diameter pellets, and subsequently heated to $1250{ }^{\circ} \mathrm{C}$ in a reducing atmosphere of $5 \% \mathrm{H}_{2}$ in $\mathrm{N}_{2}$ for approximately $96 \mathrm{~h}$. These grinding and heating steps were repeated until phase purity was achieved. A Panalytical X'Pert Pro diffractometer with a $\mathrm{Cu} K \alpha$ x-ray source was used to determine the phase purity of the samples.

Time-of-flight neutron powder-diffraction experiments observed the low-temperature nuclear structure and the long-range magnetic structure. This was completed on GEM, the General Materials diffractometer at the ISIS Neutron and Muon Source, Didcot, Oxfordshire, UK [21]. Approximately $6 \mathrm{~g}$ of powder was placed in an 8-mm cylindrical vanadium can and the data were corrected for absorption. The collected dataset can be found in Ref. [22]. Structural refinements against these data used the General Structure Analysis System (GSAS), and a convolution of the Ikeda-Carpenter and pseudo-Voigt functions to describe the peak shapes [23]. The background was modeled using a shifted Chebyshev function. The magnetic form factor of the $\mathrm{Mn}^{2+}$ ions was taken from the neutron data booklet [24].

Magnetic susceptibility measurements were carried out using a Quantum Design MPMS3 based at the Materials Characterisation Laboratory at the ISIS Neutron and Muon Source. Temperature-dependent magnetic susceptibility was measured between 2 and $300 \mathrm{~K}$ under zero-field-cooled (ZFC) and field-cooled (FC) conditions in an applied field of $1000 \mathrm{G}$. Approximately $100 \mathrm{mg}$ of $\mathrm{Ba}_{2} \mathrm{MnWO}_{6}$ was placed in a gelatin capsule and held tightly in place using a small quantity of polytetrafluoroethylene (PTFE) tape. The filled capsule was then inserted into a plastic straw for measurement.

Muon spin-relaxation measurements were carried out on EMU at the ISIS Neutron and Muon Source, where zero, transverse, and longitudinal fields were applied to the sample [25]. Around $5 \mathrm{~g}$ of $\mathrm{Ba}_{2} \mathrm{MnWO}_{6}$ was densely packed into silver foil and placed on a silver sample holder. Zero-field measurements were completed between 2 and $30 \mathrm{~K}$. A transverse field of $100 \mathrm{G}$ was applied between 2 and $30 \mathrm{~K}$ to observe the transition temperature. Longitudinal-field measurements were carried out to study the decoupling of muon spins from the internal magnetic field. The measured data can be observed using the referenced DOI [26]. The data were analyzed using the MANTID software [27].

Inelastic neutron-scattering measurements were performed on the LET time-of-flight direct geometry spectrometer at the ISIS facility of the Rutherford Appleton Laboratory [28]. The sample was contained in a cylindrical aluminum can of diameter $10 \mathrm{~mm}$ and height $30 \mathrm{~mm}$, which also contained helium exchange gas. The sample can was cooled by a helium cryostat. Data were collected using neutrons with incident energies of $3.7 \mathrm{meV}$ at temperatures between 2 and $100 \mathrm{~K}$ for $\sim 3 \mathrm{~h}$ each; these measured datasets can be found using Ref. [29]. The data were reduced using the MANTIDPLOT software package [27]. The raw data were corrected for detector efficiency and time-independent background following standard procedures [30].

\section{RESULTS}

\section{A. Magnetic properties}

DC-magnetometry data collected on $\mathrm{Ba}_{2} \mathrm{MnWO}_{6}$ indicate an antiferromagnetic transition at approximately $8 \mathrm{~K}$ with no divergence between the FC and ZFC data shown in Fig. 2. Curie-Weiss fitting of the data between 100 and $300 \mathrm{~K}$ yielded Curie and Weiss constants of $C=4.91(1) \mathrm{cm}^{3} \mathrm{~K} \mathrm{~mol}^{-1}$ and $\theta=-63(1) \mathrm{K}$. The Curie constant was used to calculate the effective magnetic moment, $\mu_{\mathrm{eff}}=6.3(3) \mu_{\mathrm{B}}$, which is close to that expected for the spin-only value of high-spin 


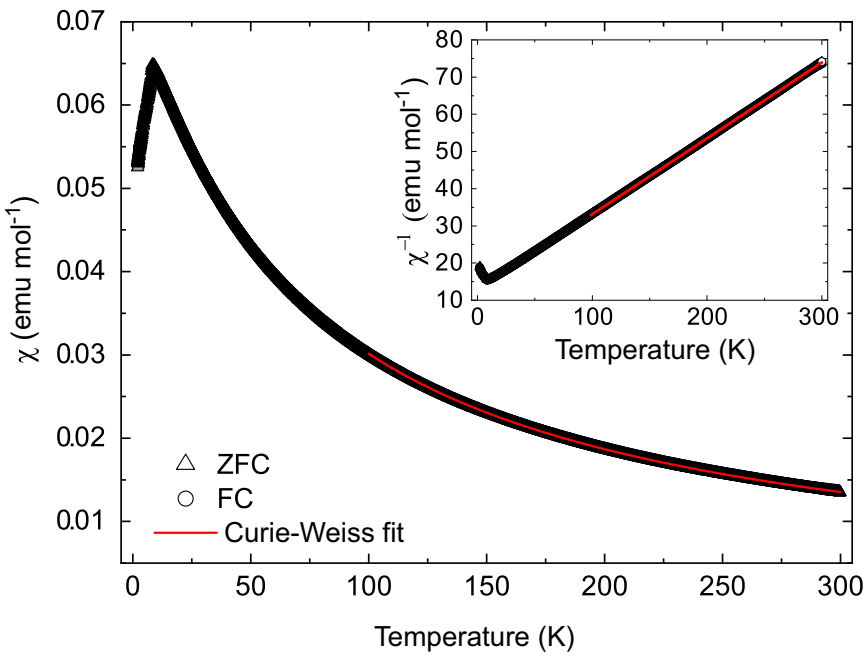

FIG. 2. DC magnetic susceptibility of $\mathrm{Ba}_{2} \mathrm{MnWO}_{6}$ measured in an applied field of $1000 \mathrm{G}$ between 2 and $300 \mathrm{~K}$. The ZFC and FC susceptibility $(\chi)$ are plotted on the main graph, while the inverse susceptibility $\left(\chi^{-1}\right)$ can be seen in the inset. No divergence is observed between the ZFC and FC responses. The susceptibility curve indicates that $\mathrm{Ba}_{2} \mathrm{MnWO}_{6}$ goes through a transition to a longrange antiferromagnetic state at $8(1) \mathrm{K}$.

$\mathrm{Mn}^{2+}$ where $\mu_{S O}=5.9 \mu_{B}$. The frustration index $(f)$, derived from the Curie-Weiss fit, $f=\theta_{\mathrm{CW}} / T_{N}=8$, indicates that $\mathrm{Ba}_{2} \mathrm{MnWO}_{6}$ is moderately frustrated, with a value below that of $f \geqslant 10$ that is considered to indicate a strongly frustrated system [1].

\section{B. Diffraction}

Laboratory $\mathrm{x}$-ray-diffraction data indicate that the singlephase double perovskite $\mathrm{Ba}_{2} \mathrm{MnWO}_{6}$ had been synthesized, with the fcc structure arising from $\mathrm{Mn}^{2+} / \mathrm{W}^{6+}$ cation ordering over the octahedral sites of the perovskite structure as previously described $[19,20]$.

Neutron powder diffraction was utilized to determine both the low-temperature nuclear structure and to analyze the magnetic structure of $\mathrm{Ba}_{2} \mathrm{MnWO}_{6}$. This was carried out between temperatures of 2 and $69 \mathrm{~K}$. Throughout the temperature range there was no peak broadening or splitting and the nuclear phase could be accurately fitted using the fcc space group, $F m \overline{3} m$, indicating no phase transition in the crystal structure down to $2 \mathrm{~K}$. Conventional thermal expansion properties were exhibited by the material; however, a change in the slope of the lattice parameter could be observed around 9(1) K which is indicative of magnetostrictive behavior [31]. A representative fitted diffraction pattern, collected at $2 \mathrm{~K}$, and the thermal evaluation of the lattice parameters are presented in Fig. 3.

In datasets collected at $T \leqslant 9 \mathrm{~K}$, a number of additional Bragg peaks were evident at higher $d$ spacings, while the data at shorter $d$ spacings remain unchanged, indicative of the onset of long-range antiferromagnetic order. These peaks were readily indexed using a doubling of the crystallographic cell along each axis corresponding to a magnetic propagation vector $\boldsymbol{k}=(1 / 2,1 / 2,1 / 2)$.
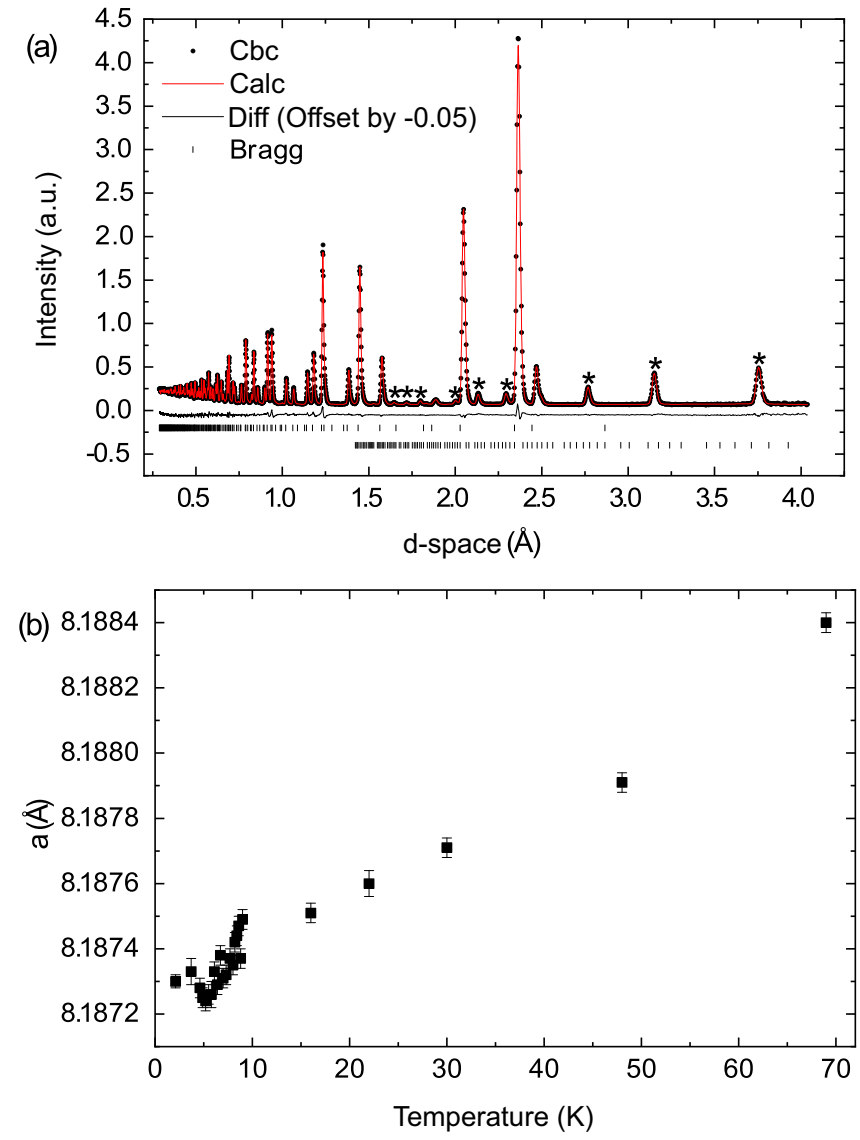

FIG. 3. (a) Neutron powder-diffraction pattern of $\mathrm{Ba}_{2} \mathrm{MnWO}_{6}$ at $2 \mathrm{~K}$. This contains both the nuclear phase (upper Bragg reflections) and the magnetic phase (lower Bragg reflections). The nuclear phase is characterized using the cubic space group $F m \overline{3} m$, with lattice parameter $a=8.18730$ (2) $\AA$. The magnetic phase has the propagation vector $\boldsymbol{k}=(1 / 2,1 / 2,1 / 2)$. The solely magnetic peaks are outlined by an asterisk. (b) Thermal evolution of lattice parameter. A general increase is observed in the lattice parameter as the temperature increases, showing conventional thermal expansion behavior.

Symmetry-allowed magnetic structures of $\mathrm{Ba}_{2} \mathrm{MnWO}_{6}$ were evaluated using representation analysis [32]. Irreducible representations of the little group $G_{k}$ were obtained for the Mn $4 a$ site $(0,0,0)$ in $F m \overline{3} m$ with $\boldsymbol{k}=(1 / 2,1 / 2,1 / 2)$ using BASIREPS [33] and SARAH [34]. Two irreducible representations were found: $\Gamma_{\mathrm{mag}}=\Gamma_{3}+\Gamma_{5}$. Both possible solutions correspond to ferromagnetic (111) planes of $\mathrm{Mn}$ moments, which couple antiferromagnetically with adjacent (111) planes. The difference between $\Gamma_{3}$ and $\Gamma_{5}$ is in the direction of magnetic moment on $\mathrm{Mn}$, which can be described as a linear combination of the basis vectors of the corresponding irreducible representation. $\Gamma_{3}$ has one basis vector such that the magnetic moment points in the [111] direction, orthogonal to the ferromagnetic (111) planes. $\Gamma_{5}$ has two basis vectors corresponding to a magnetic moment within the (111) planes.

Both magnetic structures were initially tested on FULLPROF [33], which allows the direct refinement of the basis vector coefficients. The final Rietveld refinements were carried out on GSAS [23]. $\Gamma_{3}$ fits the data poorly with $R_{\mathrm{wp}}=12.2$ and $\chi^{2}=$ 34.78 as it predicts zero intensity for many of the observed 
TABLE I. Structural parameters derived from Rietveld refinement against neutron powder diffraction collected on $\mathrm{Ba}_{2} \mathrm{MnWO}_{6}$ at $2 \mathrm{~K}$. Bond valence sums (BVS) were calculated using tabulated values [35]. Space group $F m \overline{3} m$ with $a=8.18730(2) \AA$. Ba on $(1 / 4,1 / 4,1 / 4)$ site, $\mathrm{Mn}$ on $(0,0,0)$ site, $\mathrm{W}$ on $(1 / 2,0,0)$ site, $\mathrm{O}$ on $(x, 0,0)$ site with $x=0.265193(3)$. Ordered magnetic moment on $\mathrm{Mn}^{2+}$ of $4.5(1) \mu_{B}$.

\begin{tabular}{llllc}
\hline \hline Atom & \multicolumn{1}{c}{$\mathrm{Ba}$} & \multicolumn{1}{c}{$\mathrm{Mn}$} & \multicolumn{1}{c}{$\mathrm{W}$} & $\mathrm{O}$ \\
\hline $100 \times U_{\text {iso }} / \AA^{2}$ & $0.16(1)$ & $0.26(2)$ & $0.08(1)$ & $0.392(6)$ \\
$M-\mathrm{O} / \AA$ & $2.89732(1)$ & $2.1711(3)$ & $1.9225(3)$ & \\
$\mathrm{BVS}$ & 2.32 & 2.14 & 5.98 & \\
\hline \hline
\end{tabular}

reflections including the most intense magnetic Bragg peak. The correct solution is $\Gamma_{5}$, although the direction of moment in the (111) plane cannot be determined from powder diffraction. We chose [112] for the Mn moment as has been reported for $\mathrm{MnO}$ [17], which shares the same magnetic structure. $\Gamma_{5}$ gave an excellent fit to the data as shown in Fig. 3(a) with $R_{\mathrm{wp}}=$ 4.52 and $\chi^{2}=4.73$ with the results presented in Table I. The magnetic structure is shown in Fig. 4(a). The refined moment on Mn was found to be $4.5(1) \mu_{B}$, the thermal evolution of which can be observed in Fig. 4(b).

\section{Muon spin rotation and relaxation}

The local nature of the muon spin-relaxation measurements on $\mathrm{Ba}_{2} \mathrm{MnWO}_{6}$ was used to complement the bulk techniques outlined previously. Initially, an external transverse field (TF- $\mu \mathrm{SR}$ ) of $100 \mathrm{G}$ was applied to the system and measurements were carried out between 1.6 and $70 \mathrm{~K}$ to identify the transition temperature accurately. When the sample is in a paramagnetic state, the muon spins couple to the applied transverse field resulting in a large oscillating signal as depicted in Fig. 5(a). In the magnetically ordered state, the static internal fields are much stronger than the weak applied transverse field and the oscillation is no longer observed. Thus, the asymmetry of the oscillating signal in TF- $\mu$ SR can be used to determine the transition temperature. Furthermore, the paramagnetic fraction of the sample at a given temperature can be estimated from the normalized magnitude of the oscillatory term relative to that observed in the paramagnetic regime at a high temperature of $70 \mathrm{~K}$.

Each of the TF- $\mu$ SR datasets could be fitted using the sum of an oscillating decay, an exponential decay, and a flat background. This is described by the following function:

$$
A(t)=A_{\text {osc }} e^{-\lambda_{\text {osc }} t} \cos (2 \pi f t+\phi)+A_{2} e^{-\lambda_{2} t}+A_{3},
$$

where $A_{\mathrm{osc}}, A_{2}$ and $A_{3}$ correspond to the asymmetries of the oscillating decay, the exponential decay, and the constant signal, respectively, $\lambda$ corresponds to the relaxation rate, $f$ is the frequency of the oscillation (which is dependent on the applied transverse field), and $\phi$ is the phase of this oscillation. The decaying functions were related to any possible relaxation within the system, arising due to unpaired electrons while the flat background can be attributed to large static magnetic fields along the muon's initial spin direction.

Figure 5(c) shows the thermal evolution of the oscillating signal fraction in $\mathrm{Ba}_{2} \mathrm{MnWO}_{6}$. At $2 \mathrm{~K}$, no asymmetry should
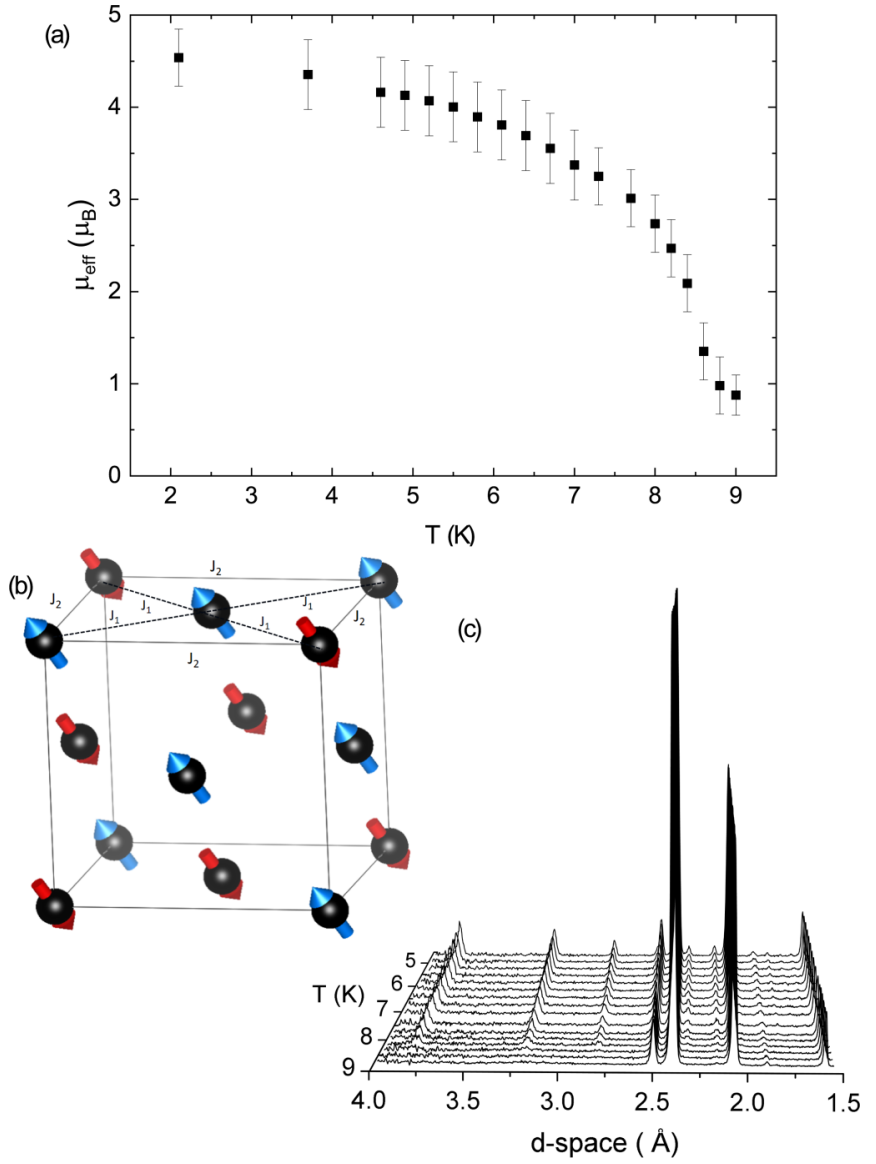

FIG. 4. (a) Schematic of the magnetic structure of $\mathrm{Ba}_{2} \mathrm{MnWO}_{6}$. Only magnetic $\mathrm{Mn}^{2+}$ ions are depicted within the crystallographic structure, with antiparallel magnetic moments outlined in red or blue. The nearest-neighbor (NN) interactions are depicted by the dashed line denoted by $J_{1}$, while the next-nearest neighbor (NNN) interactions are signified by $J_{2}$. (b) The thermal evolution of the ordered magnetic moment of $\mathrm{Ba}_{2} \mathrm{MnWO}_{6}$. This reaches a maximum value of $4.5(1) \mu_{\mathrm{B}}$ at $2 \mathrm{~K}$ and decreases as the temperature tends to $T_{\mathrm{N}}$. (c) Thermal evolution of the magnetic Bragg peaks from neutron diffraction data collected on GEM. The magnetic Bragg peaks are absent at $9 \mathrm{~K}$ and increase in magnitude as the temperature decreases.

be observed as the internal magnetic field is significantly greater than the applied transverse field. However, a small oscillation is observed which is attributed to muons implanted in the silver sample holder. As the temperature increases, the fraction remains small until $8.25(8) \mathrm{K}$ where the relaxing proportion of the material begins to increase, therefore this was identified as the transition temperature of the material. A further increase in the temperature shows a gradual increase in this relaxing proportion, and this was attributed to potential short-range magnetic correlations within the material. This gradual increase did not reach a plateau over the measured range up to $30 \mathrm{~K}$.

Zero-field measurements were carried out between 1.6 and $30 \mathrm{~K}$. These showed a characteristic drop in initial asymmetry as the sample was cooled below $T_{\mathrm{N}}$. Oscillations in asymmetry were not observed in the ordered state due to the high $\mathrm{Mn}^{2+}$ moment and limited time resolution of the instrument used. 


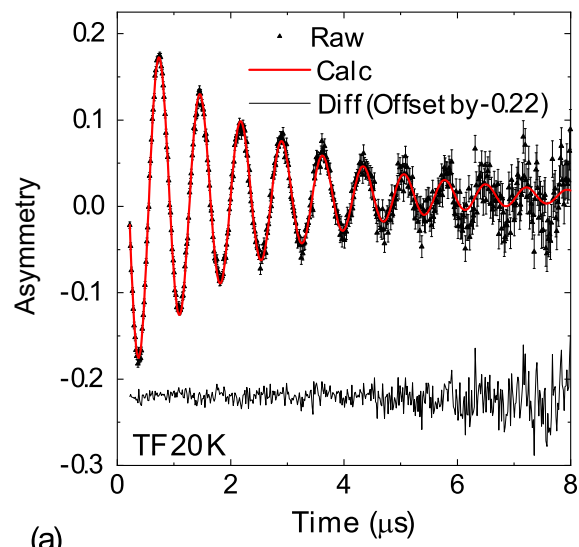

(a)

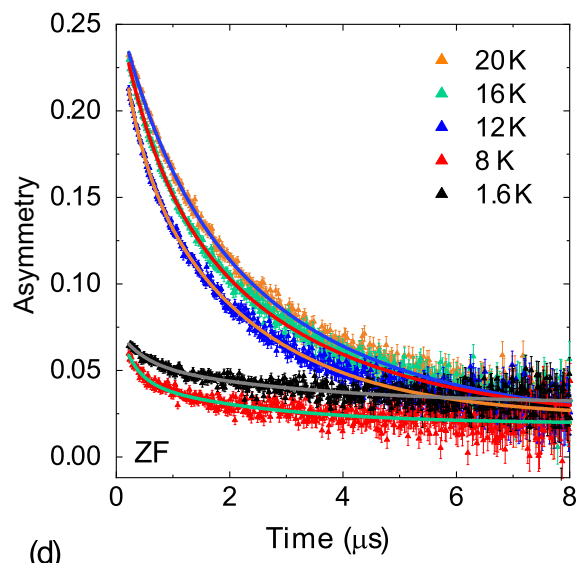

(d)

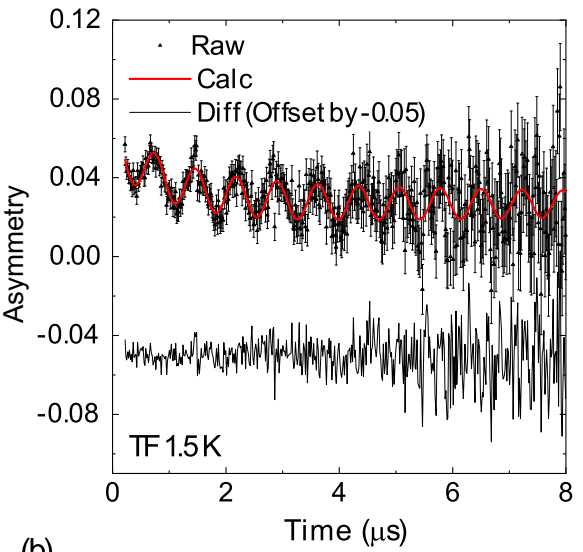

(b)

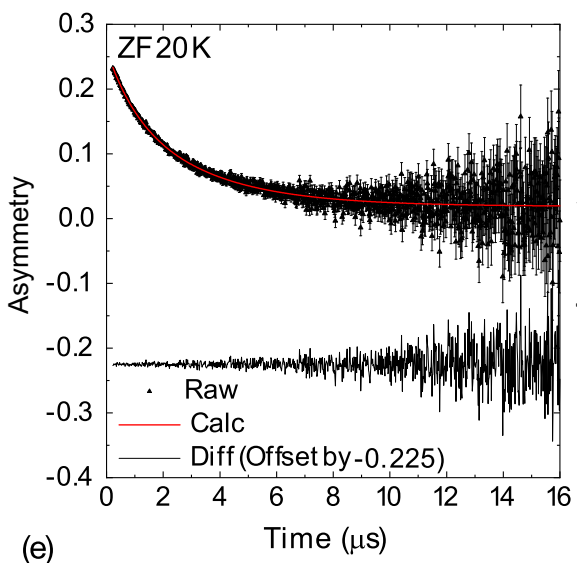

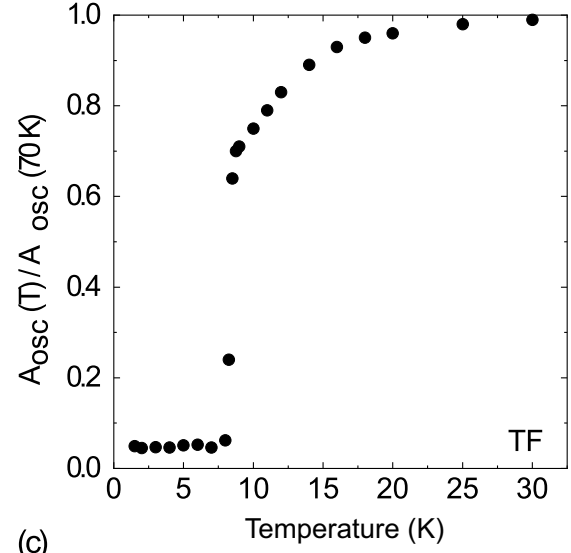

(c)

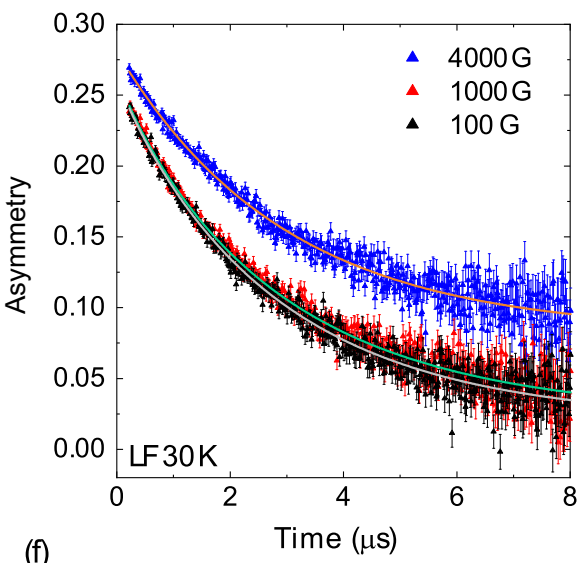

FIG. 5. (a), (b) TF- $\mu$ SR measurement carried out at 20 and $1.5 \mathrm{~K}$, respectively. At $20 \mathrm{~K}$, oscillations are observed as muons couple to the perpendicular field, indicating that the muons observe paramagnetic behavior. At $1.5 \mathrm{~K}, \mathrm{Ba}_{2} \mathrm{MnWO}_{6}$, is magnetically ordered and therefore these oscillations are reduced in amplitude as only muons stopping in the silver sample holder do not experience the large magnetic fields inside the sample. (c) The asymmetry of the oscillating relaxation was then normalized and has been plotted against temperature. It is initially low between 2 and $\sim 8 \mathrm{~K}$ before a sharp rise at the transition temperature; this is followed by a more gradual increase between 8.5 and $30 \mathrm{~K}$, which is attributed to short-range correlations. (d) ZF measurements carried out at a range of temperatures; above the transition the relaxation has a high asymmetry, while below $T_{N}$ the initial asymmetry is damped. (e) ZF measurement carried out at $20 \mathrm{~K}$, fit using $A(t)=A_{1} e^{-\lambda_{1} t}+A_{2} e^{-\lambda_{2} t}+A_{3}$. This fit is indicative of potential short-range correlations as a second exponential $A_{2}$, corresponding to slowly fluctuating dynamic fields, is necessary to fit the data. (f) The longitudinal-field datasets collected at $30 \mathrm{~K}$, which is significantly above $T_{N}=8(1) \mathrm{K}$. Full decoupling of the muon spin is not achieved even at a field of $4000 \mathrm{G}$, whereas in a paramagnetic state this should occur in low magnetic fields of up to 50-G strength. The high fields required could suggest short-range correlations between the $\mathrm{Mn}^{2+}$ cations.

Between 1.6 and $20 \mathrm{~K}$ the ZF datasets could be fitted using

$$
A(t)=A_{1} e^{-\lambda_{1} t}+A_{2} e^{-\lambda_{2} t}+A_{3},
$$

where $A_{1}$ corresponds to a slow relaxation and $A_{2}$ corresponds to a faster relaxation, with relaxation rates of $\lambda_{1}$ and $\lambda_{2}$, respectively. While Eq. (3) has the same form both below and above the magnetic transition, its physical origin is different. In the magnetically ordered state, the expected oscillations in asymmetry cannot be resolved on EMU. Instead, this component is observed as the fast exponential $A_{2}$ [36]. Unexpectedly, two exponentials are also needed to fit the data above $T_{\mathrm{N}}$ up to $20 \mathrm{~K}$. In this regime, the slow exponential $A_{1}$ corresponds to typical paramagnetic behavior. The fluctuation frequency, $v$, of the dynamic magnetic fields at the muon site is inversely proportional to the relaxation rate $\lambda$. Thus, this slow relaxation corresponds to quickly changing dynamic fields as expected in a paramagnet. The relaxation rate $\lambda_{2}$ of the fast exponential $A_{2}$ is significantly higher, corresponding to slowly fluctuating dynamic fields. This is consistent with the short-range correlated magnetic state proposed based on the TF results. At $30 \mathrm{~K}$, the function reduces to

$$
A(t)=A_{1} e^{-\lambda_{1} t}+A_{3},
$$

at which point the short-range correlations are no longer detected by the implanted muons.

Longitudinal-field (LF) measurements were completed at a variety of temperatures both below and above the transition temperature. Below $T_{\mathrm{N}}$, the muon spins could not be fully decoupled from the local magnetic fields within $\mathrm{Ba}_{2} \mathrm{MnWO}_{6}$ even in an applied longitudinal field of $4000 \mathrm{G}$, the strongest field strength available. This was expected as the material is in a magnetically ordered state and indicates that the system possesses internal magnetic fields which are stronger than the applied external field. LF- $\mu$ SR measurements carried out above the transition temperature support the presence of short-range magnetic correlations within the system. Like the 

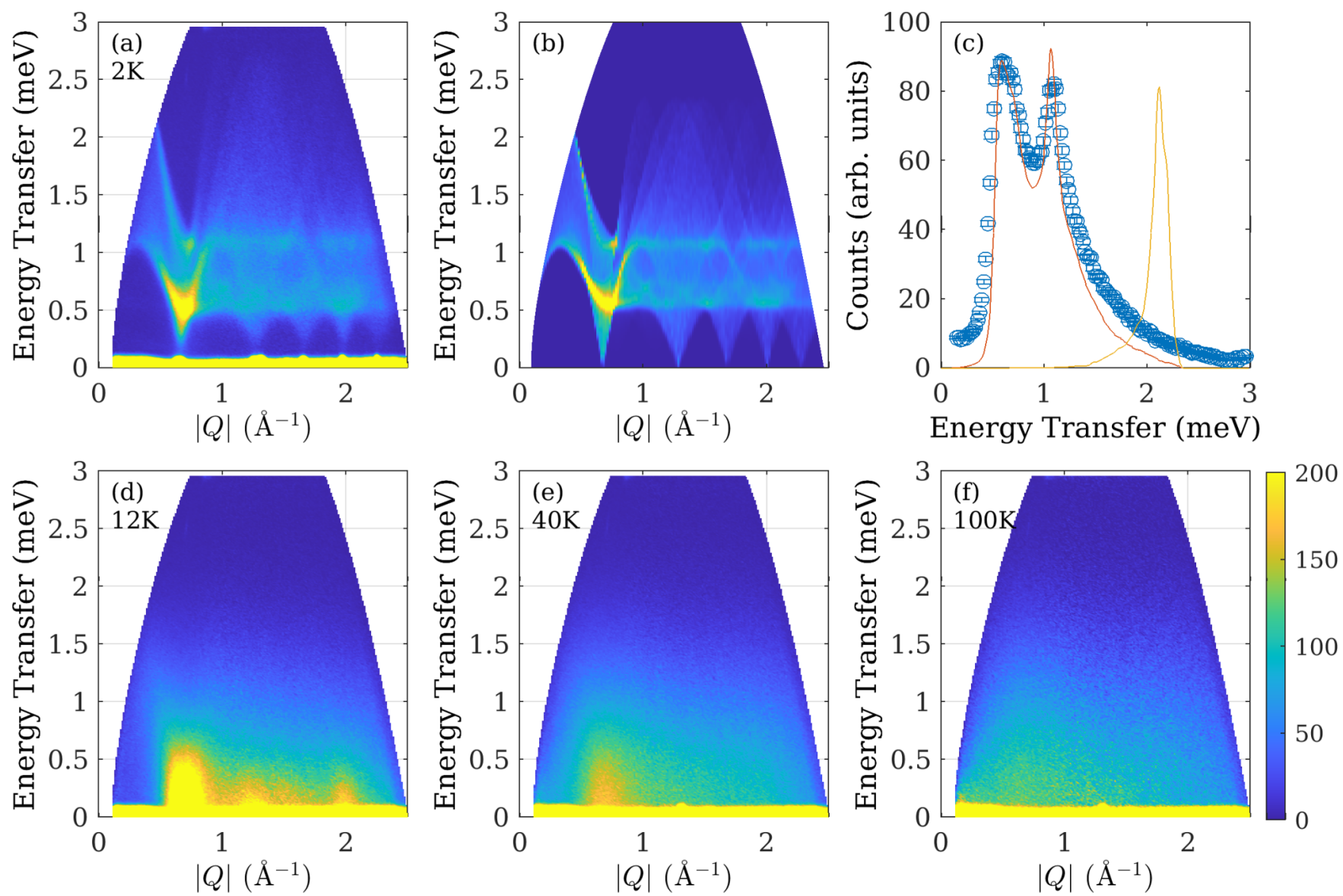

FIG. 6. (a) Inelastic neutron-scattering data collected on LET at 2 K. Distinct spin waves can be observed due to long-range order within the system. (b) A graphical output of the SpinW simulation carried out to obtain the coupling constants $J_{1}$ and $J_{2}$; these were determined to be -0.080 and $-0.076 \mathrm{meV}$, respectively, indicating antiferromagnetic interactions for both the NN and NNN interactions. Both the experimentally observed data and the simulation tally to a reasonable degree, indicating that the Heisenberg model used was consistent with the data. (c) A vertical cut through the dataset for $|Q|=0.9-1.1 \AA^{-1}$; the left-hand solid line shows the simulation output using $J_{1}=-0.080$ meV and $J_{2}=-0.076 \mathrm{meV}$, where it is clear that the peaks in the data intensity are well reproduced, while the right-hand solid line depicts only one peak in intensity which would be observed if $J_{2}=-0.010 \mathrm{meV}$ and $J_{2}=-0.146 \mathrm{meV}$. It is clear from this that it is necessary for $J_{1}$ and $J_{2}$ to have similar magnitudes, where the peak splitting determines the ratio between $J_{1}$ and $J_{2}$. (d), (e), (f) The observed inelastic neutron-scattering data collected at 12,40 , and $100 \mathrm{~K}$, respectively. These are indicative of short-range magnetic excitations being present within the system above $T_{N}$, which diminish as the temperature is increased.

lower-temperature datasets, a full decoupling of the relaxation was not achieved when a longitudinal field of $4000 \mathrm{G}$ was applied to the sample, as shown in Fig. 5(f). This suggests a strong electronic contribution to relaxation even above $T_{\mathrm{N}}$, which is consistent with a short-range correlated state. In an ideal paramagnetic state, a field of $50 \mathrm{G}$ would be enough to fully decouple the muon spins from weak nuclear magnetic fields.

\section{Inelastic neutron scattering}

Inelastic neutron-scattering measurements were carried out at a range of temperatures both below and above the transition temperature. Below $T_{\mathrm{N}}$ these could be used to quantify the magnetic coupling constants within the system. Above $T_{\mathrm{N}}$, these could be used to give firm evidence of short-range magnetic excitations taking place within $\mathrm{Ba}_{2} \mathrm{MnWO}_{6}$.

Initially, inelastic neutron scattering was carried out at $2 \mathrm{~K}$, where distinct spin waves were observed within the system.
This pattern was then compared to a SpinW simulation, based on the Hamiltonian presented in Eq. (1), to extract the values of the coupling constant using linear spin-wave theory [37]. The spin-wave spectra were well described by the $J_{1}-J_{2}$ Heisenberg model with $\mathrm{NN} J_{1}$ and $\mathrm{NNN} J_{2}$ interactions. From this practice it was found that $J_{1}=-0.080 \mathrm{meV}$, while $J_{2}=$ $-0.076 \mathrm{meV}$. Figure 6 shows the level of agreement between the measured and simulated inelastic neutron-scattering data. This results in a $J_{2} / J_{1}$ ratio of 0.95 , indicating that both are of similar strength. Despite $J_{1}$ being stronger than $J_{2}$, this $J_{2} / J_{1}$ ratio corresponds to type II magnetic order in the fcc $J_{1}-J_{2}$ model [12]. This is because in the fcc model all antiferromagnetic $J_{2}$ interactions can be satisfied simultaneously but that is not the case for $J_{1}$ interactions. When testing this model, a dominant $J_{2}$ was also investigated using $J_{1}=-0.010 \mathrm{meV}$ and $J_{2}=-0.146 \mathrm{meV}$; however, it was clear that this did not correlate with what was observed within the system. This is most clearly demonstrated by taking vertical cuts through the data as a function of energy transfer, integrating between 
$|Q|=0.9$ and $1.1 \AA^{-1}$, and comparing these with cuts through the SpinW simulations for the two different models. As depicted in Fig. 5(c), the model with a dominant $J_{2}$ is unable to reproduce the double peak structure seen in the data and the model with $J_{2} / J_{1}=0.95$.

Further inelastic neutron-scattering measurements were carried out above the transition temperature at 12, 20, and $40 \mathrm{~K}$, as shown in Fig. 5. At each of these temperatures distinct excitations could be observed which were established to be magnetic in nature as they disappeared at high temperatures and had greatest intensity at low momentum transfer. These magnetic excitations were found to originate from the same $|Q|$ positions at the elastic line as the spin waves observed from the ordered state in the $2 \mathrm{~K}$ measurement. This suggests the short-range correlations are related to the magnetic order below $T_{\mathrm{N}}$. These short-range magnetic correlations can still be observed at $40 \mathrm{~K}$, approximately five times the Néel temperature. At $100 \mathrm{~K}$, these excitations could no longer be observed, indicating that short-range correlations had been disrupted by $k_{B} T$ at this temperature.

\section{DISCUSSION}

$\mathrm{Ba}_{2} \mathrm{MnWO}_{6}$ is a frustrated fcc antiferromagnet with a frustration index of $f=8$. Each of the techniques outlined within this article show that the transition to a fully ordered antiferromagnet occurs at 8(1) K. A combination of neutron powder diffraction and inelastic neutron scattering show that $\mathrm{Ba}_{2} \mathrm{MnWO}_{6}$ displays characteristic type II antiferromagnetism where $J_{1}$ and $J_{2}$ have very similar magnitudes.

Both $J_{1}$ and $J_{2}$ interactions occur along Mn-O-W-O-Mn extended superexchange pathways with different angles. For the NN $J_{1}$ interaction this is a $90^{\circ}$ pathway, whereas the NNN $J_{2}$ interaction occurs along lattice parameter $a$ in a $180^{\circ}$ extended superexchange pathway. In the type II magnetic structure, the $J_{2}$ interactions are unfrustrated as all NNN spins couple antiferromagnetically. The magnetic frustration arises through the antiferromagnetic $J_{1}$ interactions, as the fcc structure does not allow all NN spins to align antiferromagnetically. Since $J_{1}$ is stronger than $J_{2}, \mathrm{Ba}_{2} \mathrm{MnWO}_{6}$ is relatively frustrated with $f=8$.

The strong $J_{1}$ observed in $\mathrm{Ba}_{2} \mathrm{MnWO}_{6}$ is unexpected and has not been reported in other $(\mathrm{Sr}, \mathrm{Ba})_{2} M \mathrm{WO}_{6}$ double perovskites where $M$ is a $3 d$ transition metal. $\mathrm{Ba}_{2} \mathrm{CuWO}_{6}$ and $\mathrm{Sr}_{2} \mathrm{CuWO}_{6}$ are both type II antiferromagnets which have been reported to have a dominant $J_{2}$ which is roughly 8-10 times greater than $J_{1}$ [38,39]. Furthermore, recent theoretical studies into $\mathrm{Sr}_{2} \mathrm{NiWO}_{6}$, another type II antiferromagnet, have indicated that $J_{2}$ is approximately 20 times larger than $J_{1}$, while previous experimental results suggest that $J_{2} / J_{1}$ is much higher than this at approximately $90[40,41]$. $\mathrm{Ba}_{2} \mathrm{FeWO}_{6}$ and $\mathrm{Sr}_{2} \mathrm{FeWO}_{6}$ have also been reported to adopt the type II AFM structure, although the magnitudes of $J_{1}$ and $J_{2}$ in these materials have not been reported [42]. These systems cannot be directly compared to $\mathrm{Ba}_{2} \mathrm{MnWO}_{6}$ which remains undistorted and cubic as these both experience a tetragonal distortion, crystallizing within the space group $I 4 / m$. $\mathrm{Ba}_{2} \mathrm{MnWO}_{6}$ is also an $S=5 / 2$ system compared to the $S=1 / 2$ cuprate systems and $S=1 \mathrm{Sr}_{2} \mathrm{NiWO}_{6}$. The difference in spin values and structure could result in these differences in behavior. Unfortunately, similar type II AFM undistorted materials which retain their cubic nature down to $2 \mathrm{~K}$ such as $\mathrm{Ba}_{2} \mathrm{CoWO}_{6}$ [43] often do not have reported values for $J_{1}$ and $J_{2}$. However, $\mathrm{Ba}_{2} \mathrm{NiWO}_{6}$ is a cubic example of a $(\mathrm{Sr}, \mathrm{Ba})_{2} M \mathrm{WO}_{6}$ double perovskite which has a $J_{2} / J_{1}$ ratio of roughly 80 , which is far greater than the 0.95 observed in $\mathrm{Ba}_{2} \mathrm{MnWO}_{6}[41,44]$. Again, this stark contrast may be a result of the difference between the spherical $S=5 / 2 \mathrm{Mn}^{2+}$ cation and the anisotropy arising from the $L=3$ orbital contribution of the $\mathrm{Ni}^{2+} d^{8}$ cations. This suggests a significant role for the orbital contribution to the magnetic coupling. There is a paucity of information on the coupling constants in related systems. However, it shows how unusual $\mathrm{Ba}_{2} \mathrm{MnWO}_{6}$ is and work is ongoing to determine if the strong dependence of orbital contribution persists once the site symmetry is lowered by structural distortion in $\mathrm{Sr}_{2} \mathrm{MnWO}_{6}$. Despite the differences in the magnetic ion involved in these systems, the spin value and the structure of each of these materials tend to adopt the type II AFM structure which indicates how robust this magnetic ordering type is to the observed large variations in the strength of magnetic interactions.

Muon spin relaxation carried out in transverse and longitudinal fields support the presence of a short-range correlated magnetic state above $T_{\mathrm{N}}$ in $\mathrm{Ba}_{2} \mathrm{MnWO}_{6}$. Inelastic neutron-scattering experiments confirmed the existence of this short-range correlated state, and suggested it is related to the magnetic order below $T_{\mathrm{N}}$. While the precise nature of this state is unknown at this time, it could be related to the well-known correlated states in the similar fcc antiferromagnet $\mathrm{MnO}$ [18].

A number of parallels may be drawn between $\mathrm{Ba}_{2} \mathrm{MnWO}_{6}$ and $\mathrm{MnO}$. Both materials possess $\mathrm{Mn}^{2+}$ ions, and therefore a $3 d^{5}$ electronic configuration corresponding to $S=5 / 2$. These are present in a high spin state and therefore experience no orbital contribution to their magnetic behavior. Both $\mathrm{Ba}_{2} \mathrm{MnWO}_{6}$ and $\mathrm{MnO}$ have type II antiferromagnetic order with $T_{N}=8(1) \mathrm{K}$ and $160 \mathrm{~K}$, respectively. The materials are relatively frustrated with frustration indexes $f=8$ for $\mathrm{Ba}_{2} \mathrm{MnWO}_{6}$ and $f=5$ for $\mathrm{MnO}$. Similar values have been reported for the $J_{2} / J_{1}$ ratio of both materials $(\sim 1)$, although the magnitude of $J_{1}$ is smaller than $J_{2}$ in $\mathrm{MnO}$, while the opposite is true in $\mathrm{Ba}_{2} \mathrm{MnWO}_{6}$. A lower $J_{2} / J_{1}$ ratio corresponds to a greater degree of frustration in the type II magnetic structure, which explains the higher frustration index experienced by $\mathrm{Ba}_{2} \mathrm{MnWO}_{6}$ compared to $\mathrm{MnO}$. $\mathrm{MnO}$ has also recently been reported to experience short-range magnetic correlations above its transition temperature which was evidenced by diffuse neutron scattering alongside reverse Monte Carlo simulations [18]. One large difference between the two materials is that $\mathrm{MnO}$ experiences a combined magnetic and structural transition at $T_{\mathrm{N}}$, while $\mathrm{Ba}_{2} \mathrm{MnWO}_{6}$ remains cubic down to $2 \mathrm{~K}$ with no structural transitions. This suggests that magnetoelastic coupling is weaker in $\mathrm{Ba}_{2} \mathrm{MnWO}_{6}$.

\section{v. CONCLUSIONS}

Neutron powder diffraction, DC magnetometry, muon spin-relaxation spectroscopy, and inelastic neutron scattering were used to investigate the magnetic nature of $\mathrm{Ba}_{2} \mathrm{MnWO}_{6}$. Type II long-range magnetic order was observed by all of these techniques, which indicated a transition temperature of 8(1) K. The magnetic structure was characterized and found to have propagation vector, $\boldsymbol{k}=(1 / 2,1 / 2,1 / 2)$, with the 
magnetic moments lying in the (111) planes. Inelastic neutron scattering showed that $\mathrm{Ba}_{2} \mathrm{MnWO}_{6}$ is well described by the fcc $J_{1}-J_{2}$ Heisenberg model with magnetic coupling constants $J_{1}=-0.080 \mathrm{meV}$ and $J_{2}=-0.076 \mathrm{meV}$. The similar magnitude and dominant $J_{1}$ is not observed in other related double perovskites, making this an interesting case for comparison, and may be caused by a combination of the cubic nature of $\mathrm{Ba}_{2} \mathrm{MnWO}_{6}$ and the large spin state of $S=5 / 2$. Muon spinrelaxation measurements indicated that short-range magnetic correlations take place above $T_{\mathrm{N}}$. Inelastic neutron-scattering measurements were able to confirm this, with magnetic excitations taking place at temperatures up to $40 \mathrm{~K}$, close to five times the magnetic transition temperature.

\section{ACKNOWLEDGMENTS}

The authors acknowledge the financial support provided by the Leverhulme Trust Research Project Grant No. RPG2017-109 and the Universities of Strathclyde and Sheffield. We also acknowledge and are grateful for the support given by Dr. Ivan da Silva based at the ISIS Neutron and Muon Source with the powder neutron-diffraction experiments. The ISIS Neutron and Muon Source and the Materials Characterisation Laboratory were both used extensively throughout this research and the authors are grateful for both being given access and the continuing support of the individuals within these facilities.
[1] J. E. Greedan, J. Mater. Chem. 11, 37 (2001).

[2] R. Morrow, M. A. McGuire, J. Yan, and P. M. Woodward, Inorg. Chem. 57, 2989 (2018).

[3] C. R. Wiebe, J. E. Greedan, G. M. Luke, and J. S. Gardner, Phys. Rev. B 65, 144413 (2002).

[4] A. P. Ramirez, A. Hayashi, R. J. Cava, and R. Siddharthan, Nature (London) 399, 333 (1999).

[5] A. M. Hallas, A. M. Arevalo-Lopez, A. Z. Sharma, T. Munsie, J. P. Attfield, C. R. Wiebe, and G. M. Luke, Phys. Rev. B 91, 104417 (2015).

[6] S. Nakatsuji, Y. Machida, Y. Maeno, T. Tayama, T. Sakakibara, J. van Duijn, L. Balicas, J. N. Millican, R. T. Macaluso, and J. Y. Chan, Phys. Rev. Lett. 96, 087204 (2006).

[7] O. Mustonen, S. Vasala, E. Sadrollahi, K. P. Schmidt, C. Baines, H. C. Walker, I. Terasaki, F. J. Litterst, E. Baggio-Saitovitch, and M. Karppinen, Nat. Commun. 9, 1085 (2018).

[8] M. A. de Vries, A. C. Mclaughlin, and J. W. G. Bos, Phys. Rev. Lett. 104, 177202 (2010).

[9] T. Aharen, J. E. Greedan, C. A. Bridges, A. A. Aczel, J. Rodriguez, G. MacDougall, G. M. Luke, T. Imai, V. K. Michaelis, S. Kroeker, H. Zhou, C. R. Wiebe, and L. M. D. Cranswick, Phys. Rev. B: Condens. Matter Mater. Phys. 81, 224409 (2010).

[10] M. A. McGuire and V. O. Garlea, Phys. Rev. B 93, 054404 (2016).

[11] M. Bieringer, J. R. Stewart, A. P. Grosvenor, M. Dragomir, and J. E. Greedan, Inorg. Chem. 58, 4300 (2019).

[12] N. N. Sun and H. Y. Wang, J. Magn. Magn. Mater. 454, 176 (2018).

[13] R. A. Tahir-Kheli, H. B. Callen, and H. Jarrett, J. Phys. Chem. Solids 27, 23 (1966).

[14] C. G. Shull and J. S. Smart, Phys. Rev. 76, 1256 (1949).

[15] F. Keffer and W. O’Sullivan, Phys. Rev. 108, 637 (1957).

[16] W. L. Roth, Phys. Rev. 110, 1333 (1958).

[17] A. L. Goodwin, M. G. Tucker, M. T. Dove, and D. A. Keen, Phys. Rev. Lett. 96, 047209 (2006).

[18] J. A. M. Paddison, M. J. Gutmann, J. R. Stewart, M. G. Tucker, M. T. Dove, D. A. Keen, and A. L. Goodwin, Phys. Rev. B 97, 014429 (2018).

[19] C. P. Khattak, D. E. Cox, and F. F. Y. Wang, J. Solid State Chem. 17, 323 (1976).

[20] A. K. Azad, S. A. Ivanov, S.-G. Eriksson, J. Eriksen, H. Rundlöf, and P. Mathieu, and R. Svedlindh, Mater. Res. Bull. 36, 2215 (2002).
[21] W. G. Williams, R. M. Ibberson, P. Day, and J. E. Enderby, Phys. B 241-243, 234 (1998).

[22] E. Cussen et al., Dynamic long range antiferromagnetic order in frustrated perovskites, $\mathrm{Ba}_{2-x} \mathrm{Sr}_{x} \mathrm{MnWO}_{6}$, STFC ISIS Neutron and Muon Source, doi: 10.5286/ISIS.E.RB1620380, 2016.

[23] A. C. Larson and R. B. Von Dreele, General Structure Analysis System (GSAS), Los Alamos National Laboratory Report LAUR 86-748, 2004.

[24] A. Rauch, W. Waschkowski, D. Dubbers, R. P. May, R. Cubitt, C. C. Wilson, E. Lelievre-Berna, J. R. Stewart, F. Tasset, J. Brown, R. S. Eccleston, R. Currat, J. Kulda, B. Farago, A. W. Hewat, G. J. McIntyre, C. J. Carlile, I. Anderson, A. Oed, M. Johnson, S. A. Mason, R. B. Von Dreele, H. Borner, and J. Tribolet, in Neutron Data Booklet, 2nd ed., edited by A.-J. Dianoux and G. Lander (Old City Publishing, Philadelphia, 2003), pp. 2.5-1-2.5-12.

[25] S. R. Giblin, S. P. Cottrell, P. J. C. King, S. Tomlinson, S. J. S. Jago, L. J. Randall, M. J. Roberts, J. Norris, S. Howarth, Q. B. Mutamba, N. J. Rhodes, and F. A. Akeroyd, Nucl. Instruments Methods Phys. Res., Sect. A 751, 70 (2014).

[26] E. Cussen et al., Investigating magnetic ordering in $\mathrm{Ba}_{2} \mathrm{MnWO}_{6}$ and $\mathrm{Sr}_{2} \mathrm{MnWO}_{6}$, STFC ISIS Neutron and Muon Source, doi:10.5286/ISIS.E.RB1820599, 2018.

[27] O. Arnold, J. C. Bilheux, J. M. Borreguero, A. Buts, S. I. Campbell, L. Chapon, M. Doucet, N. Draper, R. Ferraz Leal, M. A. Gigg, V. E. Lynch, A. Markvardsen, D. J. Mikkelson, R. L. Mikkelson, R. Miller, K. Palmen, P. Parker, G. Passos, T. G. Perring, P. F. Peterson, S. Ren, M. A. Reuter, A. T. Savici, J. W. Taylor, R. J. Taylor, R. Tolchenov, W. Zhou, and J. Zikovsky, Nucl. Instruments Methods Phys. Res., Sect. A 764, 156 (2014).

[28] R. I. Bewley, J. W. Taylor, and S. M. Bennington., Nucl. Instruments Methods Phys. Res., Sect. A 637, 128 (2011).

[29] H. Walker, Spin wave investigation in $\mathrm{Ba}_{2} \mathrm{MnWO}_{6}$, STFC ISIS Neutron and Muon Source, doi:10.5286/ISIS.E.RB1890354-1, 2019.

[30] C. G. Windsor, Pulsed Neutron Scattering (Taylor \& Francis, London, 1981).

[31] M. C. Viola, M. J. Martínez-Lope, J. A. Alonso, J. L. Martínez, J. M. De Paoli, S. Pagola, J. C. Pedregosa, M. T. Fernández-Díaz, and R. E. Carbonio, Chem. Mater. 15, 1655 (2003).

[32] E. F. Bertaut, Acta Crystallogr. Sect. A 24, 217 (1968). 
[33] J. Rodríguez-Carvajal, Physica B: Condens. Matter 192, 55 (1993).

[34] A. S. Wills, Phys. B Condens. Matter 276-278, 680 (2000).

[35] N. E. Brese and M. O'Keeffe, Acta. Crystallogr. B 47, 192 (1991).

[36] P. M. S. Monteiro, P. J. Baker, A. Ionescu, C. H. W. Barnes, Z. Salman, A. Suter, T. Prokscha, and S. Langridge, Phys. Rev. Lett. 110, 217208 (2013).

[37] S. Toth and B. Lake, J. Phys.: Condens. Matter 27, 166002 (2015).

[38] O. Mustonen, S. Vasala, H. Mutch, C. I. Thomas, G. B. G. Stenning, E. Baggio-Saitovitch, E. J. Cussen, and M. Karppinen, Chem. Commun. 55, 1132 (2019).
[39] H. C. Walker, O. Mustonen, S. Vasala, D. J. Voneshen, M. D. Le, D. T. Adroja, and M. Karppinen, Phys. Rev. B 94, 064411 (2016).

[40] N. Rezaei, T. Hashemifar, M. Alaei, F. Shahbazi, S. J. Hashemifar, and H. Akbarzadeh, Phys. Rev. B 99, 104411 (2019).

[41] Y. Todate, J. Phys. Chem. Solids 60, 1173 (1999).

[42] A. K. Azad, S. G. Eriksson, A. Mellergård, S. A. Ivanov, J. Eriksen, and H. Rundlöf, Mater. Res. Bull. 37, 1797 (2002).

[43] M. J. Martinez-Lope, J. A. Alonso, M. T. Casais, and T. Fernandez-Diaz, Eur. J. Inorg. Chem. 6, 2463 (2002).

[44] D. E. Cox, G. Shirane, and B. C. Frazer, J. Appl. Phys. 38, 1459 (1967). 